\title{
Phenotypic spectrum of SLC25A4 mutations (Review)
}

\author{
JOSEF FINSTERER $^{1^{*}}$ and SINDA ZARROUK-MAHJOUB ${ }^{2 *}$ \\ ${ }^{1}$ Department of Neurology, Municipal Hospital Rudolfstiftung, A-1180 Vienna, Austria; \\ ${ }^{2}$ University of Tunis El Manar and Genomics Platform, Pasteur Institute of Tunis, Tunis 1068, Tunisia
}

Received May 19, 2017; Accepted June 15, 2018

DOI: $10.3892 /$ br.2018.1115

\begin{abstract}
There is no comprehensive overview concerning the phenotypic variability in patients carrying SLC25A4 mutations available. Therefore, the aim of the present review was to summarise and discuss recent findings concerning the clinical presentation and phenotypic heterogeneity of SLC25A4 mutations. The study was conducted by systematically reviewing the literature using the search terms 'mitochondrial', "myopathy', 'nuclear DNA', 'mitochondrial DNA', in combination with 'SLC25A4' or 'AAC1'. The results indicated that the phenotypic heterogeneity in patients carrying a SLC25A4 mutation is broader than so far anticipated. Patients carrying a SLC25A4 mutation not only manifest as encephalo-myo-cardiomyopathy but also with scoliosis, cataract, depression, headache, hydrocephalus or arterial hypertension. SLC25A4 mutations may result in mtDNA depletion or multiple mitochondrial (mt)DNA deletions. SLC25A4-associated mtDNA depletion presents with the more severe phenotype and the worse outcome than patients with multiple mtDNA deletions. Depletion syndrome due to SLC25A4 mutations is associated with congenital respiratory insufficiency requiring mechanical ventilation with poor prognosis in the majority of the cases. Mutations in the SLC25A4 gene manifest phenotypically with multiorgan abnormalities in addition to encephalo-myo-cardiomyopathy. SLC25A4 mutations, causing mtDNA depletion, present with a more severe phenotype, including respiratory insufficiency and more widespread cerebral disease than mutations causing multiple mtDNA deletions.
\end{abstract}

\section{Contents}

1. Introduction

2. Search method

Correspondence to: Dr Josef Finsterer, Department of Neurology, Municipal Hospital Rudolfstiftung, Postfach 20, A-1180 Vienna, Austria

E-mail: fifigs1@yahoo.de

${ }^{*}$ Contributed equally

Key words: mitochondrial, mtDNA, myopathy, cardiac involvement, SLC25A4, central nervous system
3. Results
4. Discussion

\section{Introduction}

Mitochondrial disorders (MIDs) are due to mutations in one of the 37 genes located on mitochondrial DNA (mtDNA) and encoding for respiratory chain subunits, in non-coding regions of the mtDNA, or in one of the 1300 nuclear DNA located genes encoding for components of various mitochondrial pathways (1). The most important and most frequently affected mitochondrial pathway is the respiratory chain and oxidative phosphorylation (1). A number of proteins are required for proper function and maintenance of the respiratory chain. The majority of these proteins are nuclearly encoded. One of these genes is SLC25A4 (ANT1), which encodes for the ADP/ATP gated pore carrier (translocator) AAC1 (2). AAC1 translocates ADP from the cytoplasm into the mitochondrial matrix and reciprocally transfers ATP from the mitochondrial matrix into the cytoplasm. AAC1 represents a homodimer embedded in the inner mitochondrial membrane (3). Mutations in the SLC25A4 gene result in either multiple mtDNA deletions or in depletion of the mtDNA $(2,4)$. Secondarily, mtDNA depletion or multiple mtDNA deletions result in reduced production of ATP and thus, energy failure and cellular dysfunction. Thus, ATP may not only be insufficiently transferred into the cytosol but also produced in too low amounts to maintain physiological cellular functions. Transmission may be autosomal dominant (4) or autosomal recessive (5). Phenotypically, SCL25A4 mutations manifest as progressive external ophthalmoplegia if transmitted in an autosomal dominant fashion and associated with multiple mtDNA deletions or as familial hypertrophic cardiomyopathy and skeletal muscle myopathy if transmitted in an autosomal recessive way and associated with mtDNA depletion. The aim of this mini-review is to summarise and discuss recent findings concerning the clinical presentation and phenotypic heterogeneity of SLC25A4 mutations.

\section{Search method}

Data for the current review were identified by searches of MEDLINE, Current Contents, EMBASE, Web of Science, Web of Knowledge, LILACS, SCOPUS and Google Scholar for references of relevant articles using the search terms 
'mitochondrial', 'myopathy', 'nuclear DNA', 'mitochondrial DNA', in combination with 'SLC25A4' or 'AAC1'. Results of the search were screened for potentially relevant studies by application of inclusion and exclusion criteria for the full texts of the relevant studies. Included were randomized controlled trials, observational studies with controls, case series and case reports. Reviews, editorials and letters were not included. Only original articles about humans, in English, French, Spanish or German and published between 1966 and 2016 were included. Reference lists of retrieved studies were checked for reports of additional studies.

\section{Results}

Altogether, 10 suitable papers were identified following application of the search terms and the exclusion and inclusion criteria. In these 10 papers, 25 patients carrying a mutation in the SLC25A4 gene and manifesting with a MID have been reported (Table I). The age of these 25 patients ranged from 0 to 71 years and onset was between 0 and 48 years. In a single patient, age was not reported. A total of 11 patients were male and 13 were female (Table I). In one patient, gender was not reported (Table I). A total of 23 patients carried a point mutation, one patient a 1 bp deletion, and one a compound heterozygous mutation (Table I). In five patients, the SLC25A4 mutation resulted in mtDNA depletion and in five patients in multiple mtDNA deletions (Table I). In 15 patients, the consequences of the SLC25A4 mutation on mtDNA were not reported (Table I). A total of 22 patients manifested with myopathy. In a single patient no information about the skeletal muscles was provided. In a single patient, clinical myopathy was reported but not confirmed by electromyography. In one patient investigation of the skeletal muscle was normal. In 22 patients, the mutation manifested in the central nervous system (CNS). Muscle hypotonia was reported in six patients, elevated cerebrospinal fluid lactate in four patients, ischemic stroke in three patients, intracerebral bleeding in two, white matter lesions in two, seizures in two and in one patient each dysarthria, hydrocephalus, insomnia, inattention, mental retardation and headache (Table I) $(2,4-9)$. In two patients, the CNS was not affected and in one patient the CNS was not mentioned at all (Table I). A total of 17 patients had cardiac involvement manifesting as non-specific cardiomyopathy in all of them (Table I). In six patients, no cardiac involvement was reported. In two patients, it remained unclear whether the heart was investigated at all. Additional manifestations reported included cataracts, arterial hypertension, depression and scoliosis in one patient each (Table I). Scoliosis in patients carrying a SLC25A4 mutation may be due to hypotonia of the axial muscles. In the most recent study of seven patients carrying a SLC25A4 mutation associated with mtDNA depletion, all of them manifested with respiratory insufficiency since birth, requiring intubation and mechanical ventilation (2). Overall, SLC25A4 mutations may result from three genetic mechanisms. They may be inherited according to an autosomal dominant trait manifesting as late onset conditions, they may be inherited according to an autosomal recessive trait resulting in early onset loss of function variants, or they may be de novo mutations resulting in an infantile-onset disease.

\section{Discussion}

The review showed that the phenotypic heterogeneity in patients carrying a SLC25A4 mutation is broader than so far anticipated. Patients carrying a SLC 25A4 mutation not only manifest with encephalo-myo-cardiomyopathy but also with scoliosis, cataract, depression, headache, hydrocephalus or arterial hypertension. SLC25A4 mutations may result in mtDNA depletion or multiple mtDNA deletions. Why some mutations cause mtDNA depletion and others cause mtDNA deletions is unknown. SLC25A4 mutations associated with mtDNA depletion present with the more severe phenotype and the worse outcome than patients in whom SLC25A4 mutations are associated with multiple mtDNA deletions. Depletion syndrome due to SLC25A4 mutations may be associated with congenital respiratory insufficiency requiring mechanical ventilation with poor prognosis in the majority of the cases. $S L C 25 A 4$ mutations may additionally enhance or reduce the phenotypic expression of MIDs.

Generally, each organ or tissue can be affected in MIDs but it is often difficult to assess which affection is truly responsible for a certain clinical manifestation, such as in case of respiratory insufficiency, hyperhidrosis or arterial hypertension. Though respiratory insufficiency is the dominant feature of the phenotype in patients with mtDNA depletion syndrome due to SLC25A4 mutations it has not been determined whether respiratory insufficiency is due to affection of the primary or secondary respiratory muscles, due to exclusive affection of the CNS, including the respiratory centres in the brain stem, or due to affection of the peripheral nerves (2). In one patient, reported by Miryounesi et al (10), general muscle hypotonia suggested CNS involvement, which may be present despite normal MRI of the cerebrum (10).

Distribution of organ affection in patients with depletion syndrome and in patients with multiple mtDNA deletions due to $S L C 25 A 4$ mutations is generally not at variance. Both groups predominantly manifest in the skeletal muscles, the brain and the heart, as encephalo-myo-cardiomyopathy. However, type and severity of clinical organ manifestations is different between these two groups. Depletion syndrome has the worse prognosis, most likely due to the more severe reduction of the mtDNA amount. CNS involvement in depletion syndromes appears to be more severe and widespread as compared to patients carrying multiple mtDNA depletions (Table I). Respiratory compromise seems to occur only in depletion syndrome.

Ischemic stroke was reported in three patients $(2,4)$ but it was not specified if the ischemic lesions are attributable to atherothrombosis, to embolism or another cause. It was also not mentioned if the three patients with stroke had cardiovascular risk factors predisposing for cardiovascular events or not. One of the three patients had cardiomyopathy but it is unclear if there was any indication for atrial fibrillation or severe systolic dysfunction that could explain cerebral ischemia. Since MIDs are the disease group most frequently associated with left ventricular hypertrabeculation/noncompaction (11), and since noncompaction is frequently complicated by heart failure, ventricular arrhythmias, stroke/embolism and sudden cardiac death (12), it is essential to investigate these patients 
Table I. Phenotype of patients carrying SLC25A4 mutations.

\begin{tabular}{|c|c|c|c|c|c|c|c|c|c|c|}
\hline Age & Sex & Mutation & TOM & Onset MP & CMP & LA & RI & CNS & Other & Refs. \\
\hline Infant & $\mathrm{f}$ & c. $239 \mathrm{G}>\mathrm{A}$ & DPL & congenital & yes & yes & yes & yes & HT & (2) \\
\hline Infant & $\mathrm{m}$ & c. $239 \mathrm{G}>\mathrm{A}$ & DPL & congenital & yes & no & yes & yes & lactate $\uparrow$, stroke, SZ & (2) \\
\hline Infant & $\mathrm{f}$ & c. $239 \mathrm{G}>\mathrm{A}$ & DPL & congenital & yes & no & yes & yes & lactate $\uparrow, \mathrm{HT}, \mathrm{SZ}, \mathrm{WML}, \mathrm{HC}$ & (2) \\
\hline Infant & $\mathrm{f}$ & c. $239 \mathrm{G}>\mathrm{A}$ & $\mathrm{nr}$ & congenital & $?$ & yes & no & yes & HT, bleeding & (2) \\
\hline Infant & $\mathrm{m}$ & c. $703 \mathrm{C}>\mathrm{G}$ & DPL & congenital & yes & yes & yes & yes & HT, stroke & (2) \\
\hline Infant & $\mathrm{m}$ & c. $703 c>G$ & $\mathrm{nr}$ & congenital & $\mathrm{nr}$ & $\mathrm{nr}$ & yes & yes & lactate $\uparrow, \mathrm{HT}$ & (2) \\
\hline Infant & $\mathrm{m}$ & c. $703 c>G$ & DPL & congenital & no & no & yes & yes & lactate $\uparrow$, HT, bleeding & (2) \\
\hline $16 \mathrm{y}$ & $\mathrm{m}$ & c. $871 \mathrm{~A}>\mathrm{G}$ & $\mathrm{nr}$ & infancy & yes & no & yes & no & dysarthria, SC & (6) \\
\hline $28 \mathrm{y}$ & $\mathrm{m}$ & c. $707 \mathrm{G}>\mathrm{C}$ & $\mathrm{nr}$ & $\mathrm{nr}$ & yes & yes & yes & no & no & $(15,16)$ \\
\hline $\begin{array}{l}1-36 y \\
(n=9)\end{array}$ & $7 \mathrm{f}: 2 \mathrm{~m}$ & c. $523 \mathrm{delC}$ & $\mathrm{nr}$ & childhood & yes & yes & yes & no & insomnia, inattention, DP & $(5)$ \\
\hline $21 \mathrm{y}$ & $\mathrm{f}$ & c. $111+1 \mathrm{G}>\mathrm{A}$ & MDel & childhood & yes & yes & yes & no & mental retardation, $\mathrm{CA}$ & (7) \\
\hline $65 \mathrm{y}$ & $\mathrm{f}$ & c. $311 \mathrm{~A}>\mathrm{G}$ & MDel & age $15 \mathrm{y}$ & yes & no & no & no & stroke, aHT & (4) \\
\hline $25 \mathrm{y}$ & $\mathrm{m}$ & A123M & MDel & childhood & yes & yes & yes & no & headache & (8) \\
\hline $\mathrm{nr}$ & $\mathrm{nr}$ & c. $865 \mathrm{G}>\mathrm{A}$ & $\mathrm{nr}$ & $26 y$ & yes & $\mathrm{nr}$ & $\mathrm{nr}$ & $\mathrm{nr}$ & $\mathrm{nr}$ & (9) \\
\hline $71 \mathrm{y}$ & $\mathrm{f}$ & D104G & MDel & $48 \mathrm{y}$ & yes & yes & no & no & WMLs & (17) \\
\hline $41 \mathrm{y}$ & $\mathrm{f}$ & D104G & MDel & $33 y$ & yes & no & no & no & no & (17) \\
\hline
\end{tabular}

Infant was between 0-6 years old (2); childhood as referred to in (5,7,8). MP, myopathy; CMP, cardiomyopathy; LA, lactic acidosis; RI, respiratory insufficiency; CNS, central nervous system; y, years; f, female; m, male; TOM, type of mutation; DPL, depletion; MDel, multiple deletions; pat., patients; HT, hypotonia; SZ, seizures; WML, white matter lesions; HC, hydrocephalus; ?, clinically yes, electromyographically no; SC, scoliosis; DP, depression; CA, cataract; aHT, arterial hypertension; NP, neuropathy; HA, hypoacusis; nr, not reported.

particularly for this abnormality since it is frequently missed if somebody is not familiar with this entity.

Myopathy in patients carrying SLC25A4 mutations manifests as ptosis, ophthalmoparesis, limb weakness, or weakness of the axial muscles (6). Since most types of skeletal muscles seem to be affected, it is quite likely that also the respiratory muscles can be affected. However, this question has not been systematically investigated in these patients yet. Since MIDs may manifest with neuropathy (13) and since some of the patients had areflexia (2), it is also conceivable that the phrenic nerve or the nerves supplying the scalene and intercostal muscles are affected by the mitochondrial defect and thus contribute to the development of respiratory insufficiency. However, it is not reported in any of the studies if affected patients had undergo nerve conduction studies of the nerves supplying the limb muscles or the phrenic nerve. There are also no reports if any of the published cases underwent needle electromyography of the limb muscles, the intercostal muscles, the paravertebral muscles, or the diaphragm to demonstrate myopathic or neuropathic changes.

Cardiac involvement in SLC25A4 carriers is not well defined since the majority of the studies does not differentiate between the various types of cardiomyopathy, including hypertrophic cardiomyopathy, dilative cardiomyopathy, restrictive cardiomyopathy, noncompaction or Takotsubo syndrome. There is also no mentioning of ventricular arrhythmias or conduction defects, which is important since they may have a strong impact on the therapy and outcome of these patients. It is also essential to screen SLC25A4 patients for pulmonary hypertension since it can be a dominant feature in MID patients (14).
In addition to affection of the muscle, heart and brain, patients carrying SLC25A4 mutations may present with scoliosis (6), psychiatric abnormalities such as depression or anxiety (5), cataract (7) or arterial hypertension (4) (Table I). Unfortunately, no prospective studies over a longer period of time have been carried out to investigate the pattern of organ involvement with progression of the disease in SLC25A4 patients. If MID patients are systematically investigated for multiorgan disease, subclinical involvement in organs other than the classically affected may be detected or multiorgan disease may become clinically manifest with progression of the disease.

In conclusion, the present review indicates that mutations in the SLC25A4 gene manifest clinically not only as encephalo-myo-cardiomyopathy but also with hydrocephalus, peripheral nerve damage, psychiatric disease, arterial hypertension or scoliosis. SLC25A4 mutations, causing mtDNA depletion, present with a more severe phenotype than mutations causing multiple mtDNA deletions. Since respiratory insufficiency can be the predominant phenotypic feature particularly in congenital cases, it should be specified if respiratory dysfunction is cerebral, myopathic or neurogenic.

\section{Acknowledgements}

Not applicable.

\section{Funding}

Not applicable. 


\section{Availability of data and materials}

Not applicable.

\section{Authors' contributions}

JF designed the review, co-conducted the literature search and wrote the first draft. SZ-M co-conducted the literature search and provided critical comments to improve the draft manuscript. All authors approved the final version of the manuscript to be published.

\section{Ethics approval and consent to participate}

Not applicable.

\section{Consent for publication}

Not applicable.

\section{Competing interests}

The authors declare that they have no competing interests.

\section{References}

1. Gorman GS, Chinnery PF, DiMauro S, Hirano M, Koga Y, McFarland R, Suomalainen A, Thorburn DR, Zeviani M and Turnbull DM: Mitochondrial diseases. Nat Rev Dis Primers 2: 16080,2016

2. Thompson K, Majd H, Dallabona C, Reinson K, King MS, Alston CL, He L, Lodi T, Jones SA, Fattal-Valevski A, et al: Recurrent De Novo Dominant Mutations in SLC25A4 Cause Severe Early-Onset Mitochondrial Disease and Loss of Mitochondrial DNA Copy Number. Am J Hum Genet 99: 860-876, 2016.

3. Mifsud J, Ravaud S, Krammer EM, Chipot C, Kunji ER, Pebay-Peyroula E and Dehez F: The substrate specificity of the human ADP/ATP carrier AAC1. Mol Membr Biol 30: 160-168, 2013.

4. Park KP, Kim HS, Kim ES, Park YE, Lee CH and Kim DS: SLC25A4 and C10ORF2 Mutations in Autosomal Dominant Progressive External Ophthalmoplegia. J Clin Neurol 7: 25-30, 2011.

5. Strauss KA, DuBiner L, Simon M, Zaragoza M, Sengupta PP, Li P, Narula N, Dreike S, Platt J, Procaccio V, et al: Severity of cardiomyopathy associated with adenine nucleotide translocator-1 deficiency correlates with mtDNA haplogroup. Proc Natl Acad Sci USA 110: 3453-3458, 2013.
6. Shamseldin HE, Smith LL, Kentab A, Alkhalidi H, Summers B, Alsedairy H, Xiong Y, Gupta VA and Alkuraya FS: Mutation of the mitochondrial carrier SLC25A42 causes a novel form of mitochondrial myopathy in humans. Hum Genet 135: 21-30, 2016.

7. Echaniz-Laguna A, Chassagne M, Ceresuela J, Rouvet I, Padet S, Acquaviva C, Nataf S, Vinzio S, Bozon D and Mousson de Camaret B: Complete loss of expression of the ANT1 gene causing cardiomyopathy and myopathy. J Med Genet 49: 146-150, 2012.

8. Palmieri L, Alberio S, Pisano I, Lodi T, Meznaric-Petrusa M, Zidar J, Santoro A, Scarcia P, Fontanesi F, Lamantea E, et al: Complete loss-of-function of the heart/muscle-specific adenine nucleotide translocator is associated with mitochondrial myopathy and cardiomyopathy. Hum Mol Genet 14: 3079-3088, 2005.

9. Agostino A, Valletta L, Chinnery PF, Ferrari G, Carrara F, Taylor RW, Schaefer AM, Turnbull DM, Tiranti V and Zeviani M: Mutations of ANT1, Twinkle, and POLG1 in sporadic progressive external ophthalmoplegia (PEO). Neurology 60: 1354-1356, 2003.

10. Miryounesi M, Fardaei M, Tabei SM and Ghafouri-Fard S: Leigh syndrome associated with a novel mutation in the COX15 gene. J Pediatr Endocrinol Metab 29: 741-744, 2016.

11. Finsterer J: Cardiogenetics, neurogenetics, and pathogenetics of left ventricular hypertrabeculation/noncompaction. Pediatr Cardiol 30: 659-681, 2009.

12. Finsterer J, Stöllberger C and Towbin JA: Left ventricular noncompaction cardiomyopathy: Cardiac, neuromuscular, and genetic factors. Nat Rev Cardiol 14: 224-237, 2017.

13. Finsterer J: Inherited mitochondrial neuropathies. J Neurol Sci 304: 9-16, 2011.

14. Finsterer J and Zarrouk Mahjoub S: Pulmonary hypertension in mitochondrial disorders. Brain Dev 35: 466, 2013.

15. Körver-Keularts IM, de Visser M, Bakker HD, Wanders RJ, Vansenne F, Scholte HR, Dorland L, Nicolaes GA, Spaapen LM, Smeets HJ, et al: Two Novel Mutations in the SLC25A4 Gene in a Patient with Mitochondrial Myopathy. JIMD Rep 22: 39-45, 2015.

16. Bakker HD, Scholte HR, Van den Bogert C, Ruitenbeek W, Jeneson JA, Wanders RJ, Abeling NG, Dorland B, Sengers RC and Van Gennip AH: Deficiency of the adenine nucleotide translocator in muscle of a patient with myopathy and lactic acidosis: A new mitochondrial defect. Pediatr Res 33: 412-417, 1993.

17. Komaki H, Fukazawa T, Houzen H, Yoshida K, Nonaka I and Goto Y: A novel D104G mutation in the adenine nucleotide translocator 1 gene in autosomal dominant progressive external ophthalmoplegia patients with mitochondrial DNA with multiple deletions. Ann Neurol 51: 645-648, 2002. 